\title{
Management of Pelvic Lymphoceles AFter Radical Prostatectomy: A Multicentre Community Based Study
}

\author{
W. Y. Khoder, M.Trottmann, M. Seitz, A. Buchner, A. Stuber, S. Hoffmann, C. G. Stief, A. J. Becker \\ Department of Urology, University Hospital Munich-Grosshadern, Ludwig-Maximilians-University Munich, Munich, Germany
}

\begin{abstract}
Introduction: Pelvic lymphoceles (LC) following radical prostatectomy (LC-RP) have an incidence up to $27 \%$. LC-managements constitute $50 \%$ of surgical interventions performed in post-RP patients.

Objectives: To describe a therapeutic algorithm for LCmanagements based on a community based representative retrospective study.

Patients and methods: Multicentre data from 304 patients with LC-RP were retrospectively examined for LC-managements. RPs were performed by various surgeons from 67 urological departments. All patients had undergone 3 weeks rehabilitation in a specialized hospital where the data base was generated. Indications and results of therapeutic manoeuvres were used to develop a general concept for planning therapy decisions.

Results: Median age was 64 years. Complications occurred in 9\% (28/304) of patients. Median LC-volume was $36 \mathrm{ml}$ (range $20-1800 \mathrm{ml}$ ). There were more complications for LCs with $\geq 100 \mathrm{ml}$ volume than those $<100 \mathrm{ml}(27 \%$ versus $17 \%, \mathrm{p}=0.346)$. Conservative therapy was the standard in uncomplicated cases $(87 \%, 239$ of 276 patients), while intervention was done in 13\% (puncture and/or drainage, surgery). Surgical intervention was performed significantly more often in complicated cases $(82 \%, 23$ from 28 patients; $\mathrm{p}<0.001)$. Based on these data, LCs can be stratified into 3 groups depending on the size and clinical presentation. Therapeutic decisions were used to develop the illustrated new therapy algorithm.

Conclusions: This study based treatment algorithm provides a rationale approach with an accurate LCclassification as regard the indications and decision making for the available LC-RP-therapies. This could facilitate management decisions. Evaluation of this concept prospectively in large patient cohort is mandatory.
\end{abstract}

Key words: Lymphocele management, pelvic lymphoceles, radical prostatectomy, complications of lymphocele

\section{INTRODUCTION}

Lymphocele (LC) was first described by Mori who found large pelvic collections in patients after gynaecological tumour operations [1]. These collections were found to contain lymphatic fluid probably as a consequence of surgical dissection and inadequate closure of afferent lymphatic vessels.

This uncommon but well documented complication was also observed after renal transplantation or pelvic surgery with an incidence of up to $27 \%$ [2]. Since serosal surface of the peritoneum absorbs lymph fluid, lymphoceles are not common following intra-abdominal procedures in comparison to the extraperitoneal approach.

Although well documented, there is no established algorithm for the managements of this postoperative problem after radical prostatectomy (RP). In this study, we aim to describe an evidence based therapeutic algorism based on a community representative retrospective study of 304 patients with LC after radical prostatectomy (LC-RP).

\section{PATIENTS AND Methods}

Data from non selected 304 patients with LC-RP, over 3 years (2002-2004), were retrospectively examined for LC-managements. All patients were for 3 weeks in a specialized rehabilitation hospital, where all management decisions were taken and/or patients were referred to specialized centres. Operations were performed by various surgeons from 67 urological departments, from which 5 clinics were high volume RP-centres ( $35 \%$ of patients). LCs were diagnosed clinically, with abdomino-pelvic ultrasound or abdominal-CT, if indicated. All patients had documented non extravasation in postoperative cystogramm in their hospitals. Any clear fluid pelvic collection in absence of hematoma was considered as LC. Decisions, indications and results of each therapeutic manoeuvre in this patients' population were used to develop a concept for therapy decisions of LC-RP. Moreover, extensive search of PUBMED database has been performed to benefit from previous experiences in optimizing this developed concept. All intervention techniques were done as described in literature (mentioned later)

For comparison of LC-volume between different patient groups (with/without complications) the Mann-Whitney U test was used, while the Wilcox matched pairs test for comparison between different times. Categorized data were analysed with the chisquare test. P-values below 0.05 were regarded as significant. All calculations were performed using the software STATISTICA (release 8, StatSoft Inc., Tulsa, $\mathrm{OK})$. 


\section{RESULTS}

Median age was 64 years with median Body Mass Index of $26.0 \mathrm{~kg} / \mathrm{m}^{2} .25 .7 \%$ of the patients had previous abdominal operations. Median prostate volume was $50 \mathrm{ml}$. RP was done in $90.5 \%$ (275 out of $304 \mathrm{pa}-$ tients), laparoscopic extraperitoneal prostatectomy (EERPE) in $7.2 \%$ (22 of 304) and perineal prostatectomy in $2.3 \%$ (7 of 304 ) of cases. There was no correlation between the localisation of the LC ( $2 \%$ left side, $57 \%$ right side, $11 \%$ bilateral, $6 \%$ paravesical) and the operation technique $(\mathrm{p}=0.390)$. Lymphadenectomy was done in $96 \%$ of these patients with a median number of 10 lymph nodes. Sentinel lymph node dissection was performed in 13 out of 304 patients $(4.3 \%)$.

Complete LC-volume data over the whole 3 weeks follow up was available from 68 patients. Median LCvolume was $36 \mathrm{ml}$ (20 to $1800 \mathrm{ml}$ ). Complete volume chart data over the 3 weeks hospitalisation period were available from 41 patients. There was a decrease in LC-size during these 3 weeks in 76\% (31 out of 41 pa- tients), an ongoing increase in 17\% (7 of 41) and no change in $7 \%$ (3 of 41) of the patients. This decrease in LC-size during the 3 week hospitalisation was found to be significant $(p=0.002)$. Regarding the maximum volume, LCs $<100 \mathrm{ml}$ occurred in $68 \%$ (46 of 68) of patients and LCs $\geq 100 \mathrm{ml}$ in $32 \%$ (22 of 68 ), respectively. The LC-volumes were higher in patients with complications than in those without complications (median volume $72 \mathrm{ml}$ versus $31 \mathrm{ml} ; \mathrm{p}=0.052$ ). Patients with $<100 \mathrm{ml} \mathrm{LC}$-volume had $17 \%$ incidence of complications (6 of 22) versus $27 \%$ in patients with $\geq 100 \mathrm{ml}$ LC-volume (8 of 46; $\mathrm{p}=0.346$ ) (Fig. 1, 3).

Complications were observed in 9\% (28 of 304) of LC-patients. Lower limbs oedema occurred in $4.3 \%$, pain in $3.0 \%$, thrombosis in $1.3 \%$, infection in $1.3 \%$ and compression of the bladder with progressively increasing incontinence in $0.3 \%$. There was no correlation between the incidence of complications and the operation technique ( $\mathrm{p}=0.393)$ (Fig. 2).

Conservative therapy was the standard in uncomplicated cases $(87 \%, 239$ of 276 Pat.) while intervention was done in $13 \%$ (37 of 276 ). In the other hand, this

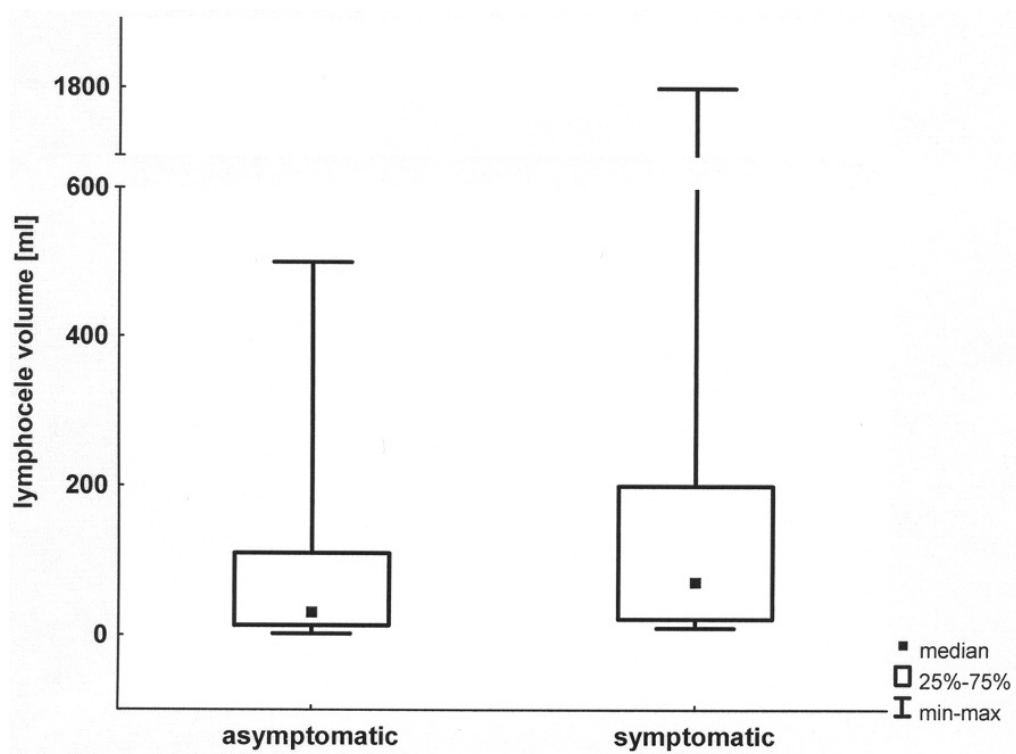

Fig. 1. Diagram showing the relation between lymphocele volumes and its symptoms/complications. Note the statistically relevant cut off value of $100 \mathrm{ml}$ volume.

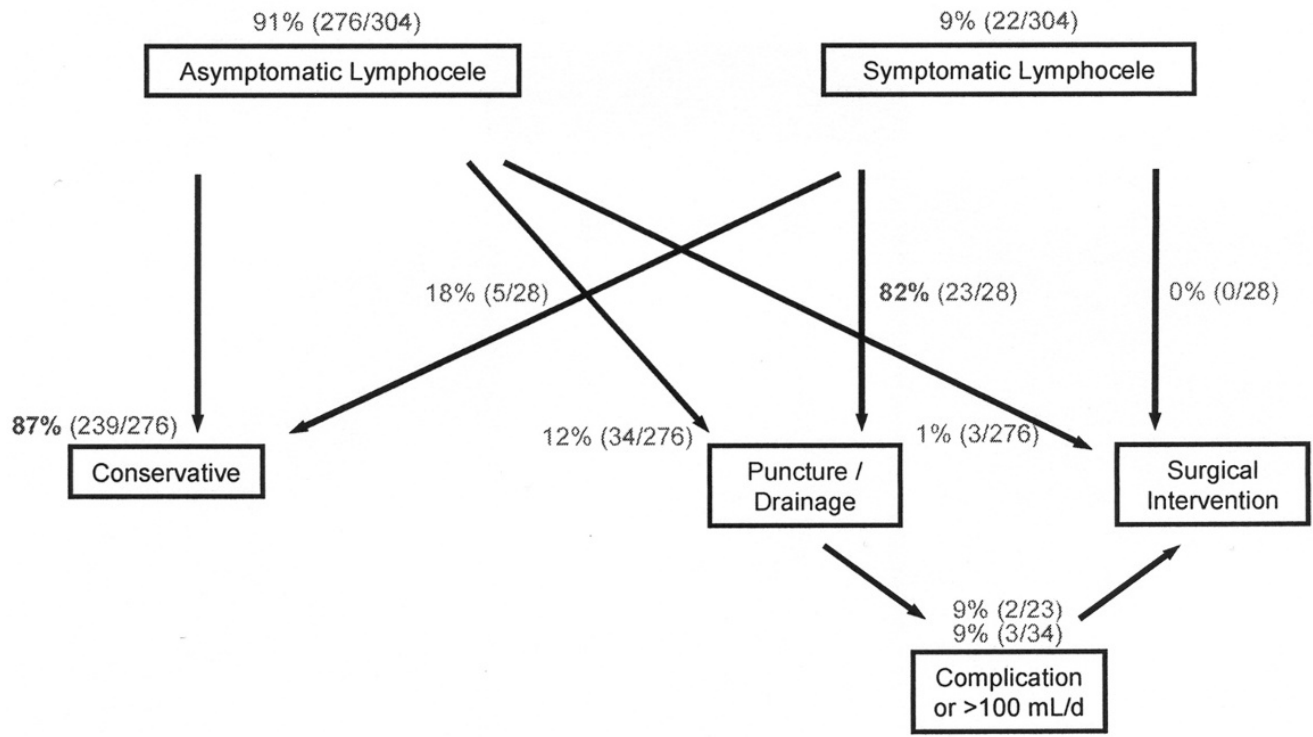

Fig. 2. Diagrammatic illustration of the managements in 304 lymphocele patients after radical prostatectomy according to the presenting clinical picture. 


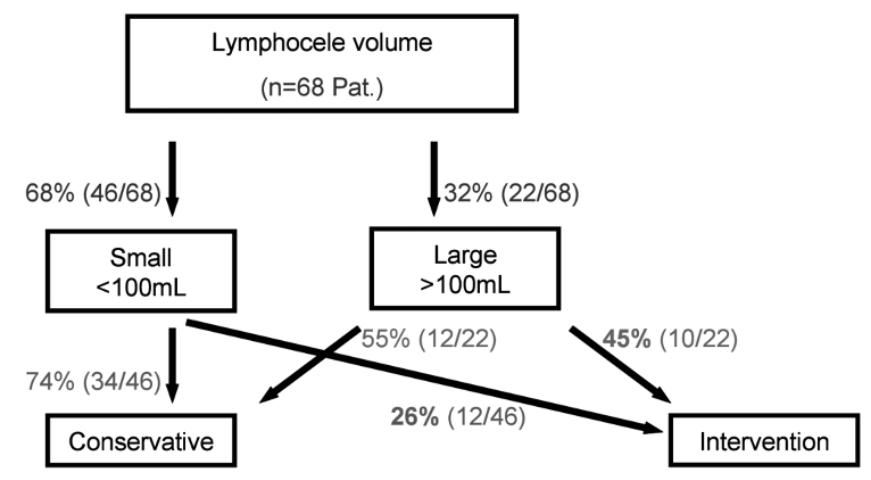

Fig. 3. Relation of lymphocele volumes and its managements. Note that with increased volumes above $100 \mathrm{ml}$, there was a statistically significant complication and intervention rates ( $26 \%$ und $45 \%$ respectively).

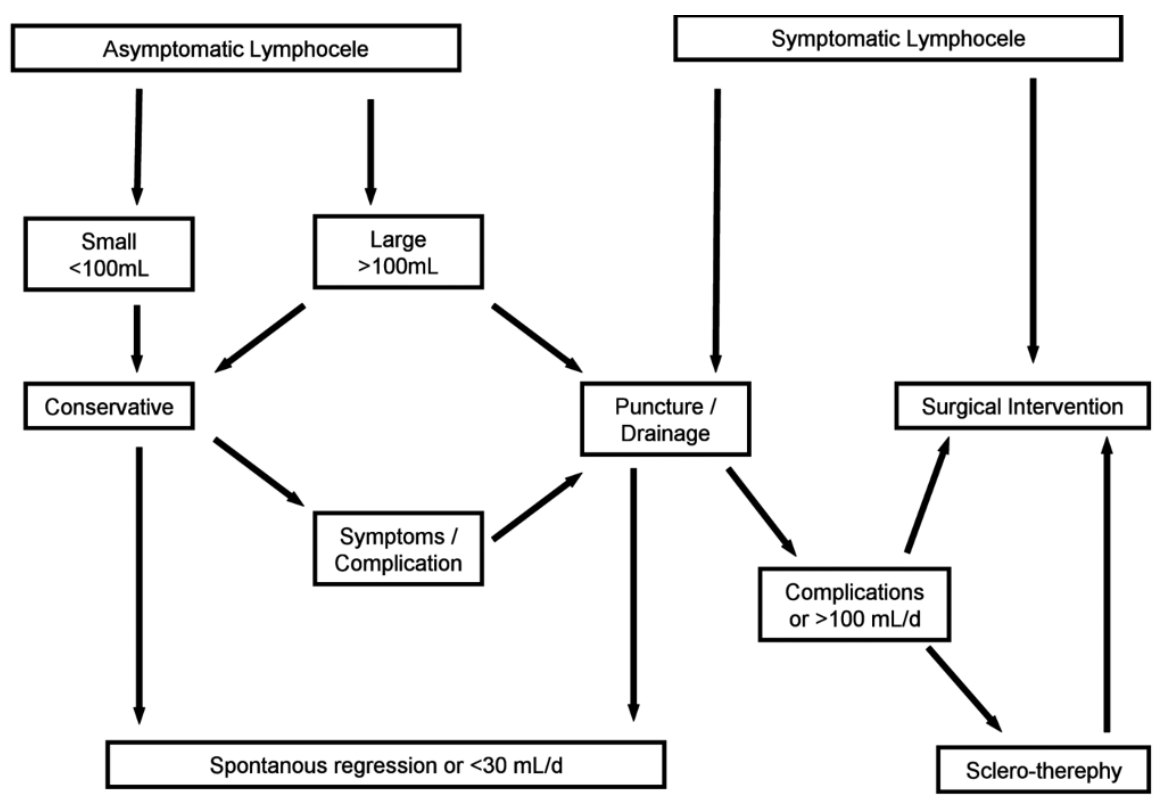

Fig. 4. Therapeutic Algorithm for pelvic lymphoceles after radical prostatectomy. conservative therapy was done only in $18 \%$ (5 of 28 ) of symptomatic LCs cases versus $82 \%$ (23 of 28 ) for intervention $(p<0.001)$ (Fig. 2).

Therapeutic intervention was done in $26 \%$ of LCcases with $<100 \mathrm{ml}$ volume (12 out of 46 patients) and in $45 \%$ of cases with $\geq 100 \mathrm{ml}$ volume (10 out of 22 patients, $\mathrm{p}=0.110$ ) (Fig. 3).

Puncture and/or drainage were done in 92\% (34 out of 37 ) of the asymptomatic patients that had a therapeutic intervention, mainly for large lymphoceles $(\geq 100 \mathrm{ml})$ as follows; puncture in $76 \%$ (26 cases), drainage in $12 \%$ (4 cases), puncture and drainage in $12 \%$ (4 cases). The remaining $8 \%$ (3 out of 37 ) asymptomatic patients with intervention had primary surgery. On the other hand, all the 23 symptomatic patients had puncture or drainage (puncture in 43\% (10 cases), drainage in 35\% (8 cases), puncture and drainage in $22 \%$ (5 cases)). Success was generally defined as $\leq 30 \mathrm{ml} /$ day for 5 days while failure $(>100$ $\mathrm{ml} /$ day/ 5 days) was managed either with sclerotherapy or surgical intervention.

Surgical intervention (peritoneal marsupialisation) after puncture and/or drainage was done in 9\% (3 out of 34) of the asymptomatic patients and in 9\% (2 out of 23) of the symptomatic patients, respectively (Fig. 2). Open surgery was done only in patients with a past history of abdominal operations or when the surgeons were not familiar with laparoscopy. Previous puncturing and/or drainage were done mainly to drain infection or to decompress the vessels.

\section{DisCUSSION}

An important relevant consequence of LC-RP is the significantly high incidence of re-intervention. Approximately $50 \%$ of all re-interventions performed in RP-patients were for LC-managements [3]. The current study is a cross sectional study. All patients underwent rehabilitation in a specialized hospital which is a routine follow up of German RP-patients. This patients population represents the community in Bavaria because it corresponds to a randomly selected group of patients from all patients who underwent radical prostatectomy in the given period (3 years). Furthermore, included patients represent small and high volume clinics, which is a further aspect of a community representative study.

Being aware that an algorithm for LC-treatment is not described in literature, we had used our mentioned retrospective data as basic resource. Therapy decisions and procedures were taken from many urologists and clinicians with different training concepts. This was used to develop an algorithm for LC-treatment (Fig. 4). 
Clinically relevant LC-characters were the volume, time of occurrence, progress and presence of complications. Interestingly, there was increase in LC-size over the first postoperative 2 weeks which became constant till 50th day in $7 \%$, decreased in $76 \%$ and continued to increase in $17 \%$. Accordingly, we propose that the earliest time to plan an elective intervention in uncomplicated cases could be one month postoperatively.

Asymptomatic lymphoceles were divided depending on its volume into 2 groups (cut off $100 \mathrm{ml}$ ). This seems logic depending on our observation that there was increase in the complication rate in LC-sizes $\geq 100 \mathrm{ml}$ ( $27 \%$ versus $17 \%$ for smaller volumes) as well as the increased rate of interventions in this group (45\% versus $26 \%$ ). This was also proved statistically (Fig. 1).

Conservative therapy was the rule for both asymptomatic groups $(87 \%)$. It was indicated for most of 1 st group, while was only applied for slowly progressive LCs or LCs lying away from iliac vessels and bladder (deep or anterovesical) in 2nd one. This confirms the published data that only small portion of LC-patients requires intervention. Subclinical-LCs (asymptomatic) occur with a much greater frequency but seldom become symptomatic requiring treatment. Rather, they resolve spontaneously [4]. Although results of conservative therapy are satisfactory, clinical suspicion should remain high in order to detect and properly treat symptomatic-LCs when they occur.

In the second group there was a high rate of puncture $(41 \%)$ and drainage $(5 \%)$. There was also a higher complication rate $(27 \%)$. Considering this high intervention/complication rates, it seems wise to puncture these LCs as a standard management (excluding the above mentioned exceptions). Furthermore, there are no obvious indications mentioned in literature for the single or recurrent percutaneous aspiration of LCfluid [5] or percutaneous drainage [6]. Whereas, indications from current study were large LCs (2 $2^{\text {nd }}$ group), rapidly accumulating LCs, symptomatic-LCs under conservative therapy or occurrence of complications.

Puncture failure was defined as leakage $\geq 100 \mathrm{ml} /$ day for 5 successive days. This definition seems logic and clinically applicable especially when sclerotherapy is planed. So far, there is no mentioned generalized definition for this in literature. Varga et al [8] considered lymphroe $\geq 100 \mathrm{ml} /$ day for one week an indication for laparoscopic LC-marsupialisation. There are no known limits for interval before surgery but in our own experience there were scant improvement after 3 weeks in resistant cases. So we recommend rash intervention if puncture and/or sclerotherapy fails.

For LC-puncture failure, following the previous definition, there were two $2^{\text {nd }}$-line therapies either sclerotherapy with many materials comparable with literature $[4,7]$ or surgical intervention [6]. Although sclerotherapy had a low success rate [4], some urologists continue to recommend multiple sittings to improve the results. A $2^{\text {nd }}$ trial sclerotherapy remains controversial without obvious indications neither in our study nor in literature. However, this seems only logic when the leakage is decreasing or dramatically de- creased in response to the $1^{\text {st }}$ sitting. Meanwhile, we believe that a trial of sclerotherapy is not a choice for high risk patients (e.g. past history of deep venous thrombosis (DVT), pulmonary embolism or sepsis). In these patients surgical intervention should be the rule to avoid further risks.

LC-development could have health relevant sequelae like secondary infection, thromboembolic events due to compression of pelvic vessels, DVT and/or pulmonary embolism [13]. Symptomatic and complicated LCs were included in one $\left(3^{\text {rd }}\right)$ group as they needed in $82 \%$ of cases the same management which was surgical intervention. Conservative therapy was only considered in $26 \%$ of these patients mostly for Painful-LCs without any compression risks. Puncture was done at first in 3 patients to decompress the vessels or to drain suspected infection. These were either prophylactically or therapeutically marsupialized afterwards. This high rate of interventions in $3^{\text {rd }}$ group $(82 \%)$ suggests that surgical intervention could provide the standard approach to avoid more complications or to prevent their occurrence.

As stated in literature, surgical drainage gives $50-70 \%$ success and $>90 \%$ success was reported after peritoneal marsupialisation [6]. Disadvantages of open technique included the requirement for general anaesthia, longer hospitalization, and surgical trauma. Recently, laparoscopic peritoneal drainage has been moving into the focus of attention [9-12]. Because of its efficacy and low morbidity, laparoscopic marsupialization is considered the first-line treatment for pelvicLCs, whenever surgery is indicated, with a success rate duplicating the open approach. Open surgery remains indicated in small, deep or extremely lateral symptomatic-LCs which are difficult to distinguish from iliac vessels or patients with extensive adhesions/bowel interposition.

Lastly, care should be exerted to treat LC-complications at first e.g. puncture for infection (or abscess formation), heparenisation and exclusion of unstable thrombus for DVT and thrombolytic therapy for pulmonary embolism. Protracted therapy or re-collection of LC may have dangerous consequences in these patients. As mentioned, these patients ( $3^{\text {rd }}$ group) are candidate for surgical intervention either as an elective or prophylactic manoeuvre.

One limitation of current study is its retrospective character, however, any prospective study will have ethical problems. Our intention was to develop a concept for future prospective evaluation which we are currently doing. Many centres and surgeons with different expertise were involved which may bias the concept. Some decisions were just one surgeon trend. But in the other hand, this had widened the concept to be a community based one. The number of patients' cohort was not too large, but from our point of view, was enough to develop a preliminary Algorithm. Finally, it would be of great help if we had a longer follow up revealing more details about our own success rates of the applied therapies. In this matter we had used the available literature. We hope to report the prospective evaluation of this algorithm in larger patients' cohort in a due time. 


\section{CONCLUSIONS}

The provided treatment algorithm is based on a retrospective multicentre study involving accurate classification, indications and success rates of the available therapies for LC-RP. It provides accurate generalized approach that could facilitate management decisions. Potential advances may include validation in larger patient cohorts.

Conflicts of interest: I disclose any commercial association that might pose a conflict in connection with my submitted article.

\section{REFERENCES}

1. Mori N. Clinical and experimental studies on the socalled Lymphocyst, which develops after radical hysterectomy in cancer of uterine cervix. J Jpn obstet Gynacol Soc 1995;2: 178-203

2. Pepper RJ, Pati J, Kaisary AV. The incidence and treatment of lymphoceles after radical retropubic prostatectomy. BJU int. 2005; 95: $772-5$

3. Augustin H, Hammerer P, Graefen M, Palisaar J, Noldus J, Fernandez S, et al: Intraoperative and perioperative morbidity of contemporary radical retropubic prostatectomy in a consecutive series of 1243 patients: results of a single centre between 1999 and 2002. Eur Urol 2003; 43: 113-5.

4. Seitz, M., Schlenker B., Stief CG. Postoperative Complications. In: Hohenfellner, M., Santucci, R. A. (Eds.) Emergencies in Urology. Heidelberg, New York: Axel Springer Verlag; 2007: 364-429.

5. Zanetta G, Trio D, Lissoni A, Dalla Valle C, Rangoni G, Pittelli M, et al. Early and short term complications after US guided puncture of gynaecologic lesions: evaluation after 1000 consecutive cases. Radiology 1993; 189; 161-4

6. Kim JK, Jeong YY, Kim YH, Kim YC, Kang HK, Choi HS. Postoperative pelvic lymphocele: treatment with simple percutaneous catheter drainage. Radiology 1999; 212 : 390-4
7. Gilliland JD, Spies JB, Brown SB, Yrizarry JM, Greenwood LH. Lymphoceles: percutaneous treatment with povidone-iodine sclerosis. Radiology 1989; $171: 227$

8. Varga Z, Hegele A, Olbert P, Hoffmann R, Schrader AJ. Laparoscopic peritoneal drainage of symptomatic lymphoceles after pelvic lymph node dissection using methylene blue instillation. Urol Int 2006;76; 335-8

9. Doehn C, Fornara P, Fricke L, Jocham D: Laparoscopic fenestration of post transplant lymphoceles. Surg Endosc 2002;16: 690-5.

10. Schilling M, Abendroth D, Kunz R: Treatment of lymphocele in renal transplant recipients by laparoscopic fenestration after transcutaneous staining. Br J Surg 1995; 82; 246-8.

11. Fallick ML, Long JP: Laparoscopic marsupialization of lymphocele after laparoscopic lymph node dissection. J Endourol 1996;10; 533- 4.

12. Iselin CE, Rochat CH, Morel P, Merlini M: Laparoscopic drainage of postoperative pelvic lymphocele. Br J Surg 1994; 81; 274-5.

13. Musch M, Klevecka V, Roggenbuck U, Kroepfl D. Complications of Pelvic Lymphadenectomy in 1,380 Patients Undergoing Radical Retropubic Prostatectomy Between 1993 and 2006. J Urol 2008;179: 923-9

Received: January 3, 2011 / Accepted: April 26, 2011

Address for correspondence:

Dr. W. Khoder, MSC, MD, FEBU

Urologische Klinik und Poliklinik

Klinikum Grosshadern

Marchioninistraße 15

81377 Munich

Germany

Tel.: +49 89-70950 (ext. Dr. Khoder)

Fax: +49 89-7095 5644

E-mail: khoder.wael@med.uni-muenchen.de 